Relations industrielles

Industrial Relations

\title{
Report on Social Security for Canada 1943, by Léonard Marsh, Toronto, University of Toronto Press, 1975, 330 pp.
}

\section{Bertrand Belzile}

Volume 32, numéro 1, 1977

URI : https://id.erudit.org/iderudit/028777ar

DOI : https://doi.org/10.7202/028777ar

Aller au sommaire du numéro

Éditeur(s)

Département des relations industrielles de l'Université Laval

ISSN

0034-379X (imprimé)

1703-8138 (numérique)

Découvrir la revue

Citer ce compte rendu

Belzile, B. (1977). Compte rendu de [Report on Social Security for Canada 1943,

by Léonard Marsh, Toronto, University of Toronto Press, 1975, 330 pp.]

Relations industrielles / Industrial Relations, 32(1), 148-149.

https://doi.org/10.7202/028777ar

Tous droits réservés @ Département des relations industrielles de l'Université Laval, 1977
Ce document est protégé par la loi sur le droit d'auteur. L'utilisation des services d'Érudit (y compris la reproduction) est assujettie à sa politique d'utilisation que vous pouvez consulter en ligne.

https://apropos.erudit.org/fr/usagers/politique-dutilisation/ 
254\%. Le tiers seulement de cette croissance s'explique par une augmentation du volume des services; l'inflation des prix des services de santé a été de $23 \%$ supérieure à celle des autres prix. La part du PNB allouée à la santé est passée de $5.3 \%$ en 1960 à $6.9 \%$ en 1973. Signalons aussi que les revenus des médecins ont crû de $332 \%$ entre 1950 et 1973 alors que l'accroissement moyen de tous les revenus d'emploi était de $192.5 \%$ pour la même période. Ce sont peut-être ces derniers chiffres qui orientent les économistes d'abord vers l'examen des complexes questions $\mathrm{d}$ paiement des services médicaux et de la rémunération des producteurs de soins.

L'économique de la santé constitue un champ d'analyse en plein développement. Le secteur de la santé est régi par des règles qui lui sont bien particulières et les modèles d'analyse économique traditionnels parviennent mal à en expliquer le fonctionnement. En effet, en matière de soins de santé, ce sont les producteurs qui définissent à la fois l'offre et la demande de services; l'ignorance relative du consommateur de même que leur situation de monopole, (corporatisme professionnel), font des médecins et d'autres producteurs de soins des agents économiques moins soumis aux règles du marché. Les prévisions et les contrôles sont donc rendus extrêmement difficiles à établir dans ce secteur et les économistes sont obligés d'inventer des instruments d'analyse adaptés à cette réalité spécifique.

Cette première conférence se veut justement un premier pas dans cette direction.

Gilles DUSSAULT

Université Laval

Report on Social Security for Canada 1943, by Léonard Marsh, Toronto, University of Toronto Press, 1975, $330 \mathrm{pp}$.

Tout historien de la sécurité sociale ne peut certes pas ignorer ce Rapport qui proposait en 1943 un système de sécurité sociale grandement amélioré pour le Canada. Avec le Rapport Marsh, on assiste à un éveil intéressant de la conscience collective face aux risques tels que la maladie, l'invalidité et le chômage, en ce sens que nos gouvernements se penchent activement sur les moyens, surtout financiers, de protéger les citoyens contre de tels risques.

La crise économique des années 30, avec toutes ses misères, a sans doute suscité cette prise de conscience, mais c'est pro- bablement la guerre 39-45 qui en est avant tout responsable. La guerre apporte avec elle une certaine prospérité. De plus, pendant une telle période, l'État offre une sécurité économique, par exemple, aux blessés de guerre et aux familles des soldats décédés. Ceci n'est pas sans inciter les gouvernants à en faire autant pour les autres citoyens. Quoiqu'il en soit, la lecture d'un tel texte amène le citoyen de 1977 à se replonger dans l'atmosphère qui régnait au début des années 40 , dans le domaine de la sécurité sociale, et lui permet d'évaluer les progrès accomplis depuis.

L'auteur nous en facilite d'ailleurs la tâche en exposant, dans une assez longue introduction écrite spécialement pour la présente édition, les grandes lignes de la perspective socio-historique de la législation sociale au moment où le Rapport a été écrit. Il y compare, par exemple, la situation canadienne avec celle qui prévalait aux États-Unis et en Grande-Bretagne.

Dans une première Partie, l'auteur fait d'abord état de l'ampleur de l'insécurité économique à l'époque et des raisons qui militent en faveur de l'assurance sociale. Il présente ensuite les principaux risques sociaux pertinents et parallèlement les principales protections possibles. Puis, après avoir défini les standards minima de protection, il les compare avec ceux existant dans la législation du moment. Enfin, dans une section traitant des principes de l'assurance sociale, il est intéressant de lire un passage où l'auteur laisse entendre que les causes premières de la pauvreté reposeraient dans la nature du système économique, ou la désorganisation sociale, et du marasme international.

Les trois parties suivantes du Rapport traitent respectivement de l'emploi, des risques universels (maladie, invalidité et vieillesse) et des besoins familiaux. L'importance accordée à l'emploi s'explique probablement par la crainte des difficultés de l'après-guerre, dans ce domaine, sans parler bien sûr de l'importance des revenus de travail dans une période où les paiements de transfert sont très faibles relativement parlant. Dans la même veine, l'auteur consacre quelque cinq pages à ce qu'on appellerait maintenant les politiques de maind'œuvre: placement, orientation et formation. Il est normal de trouver aussi une section sur l'assurance-chômage dont le premier régime date de 1940 (si on exclut la tentative de 1935-37). Une dernière section de cette partie traite de l'assistancechômage, car, entre autres raisons, il ne faut pas oublier que l'assurance couvrait moins de travailleurs que maintenant. Il est 
opportun de noter que c'est seulement en 1956 que le Gouvernement fédéral édictait sa Loi de l'assistance-chômage en vertu de laquelle il verserait aux provinces des sommes appréciables en fonction des chômeurs difficilement employables.

Puis, l'auteur couvre longuement, dans une troisième partie, les risques universels déjà mentionnés, soit la maladie, l'invalidité et la vieillesse. Déjà, les accidents du travail étaient assurés dans une certaine mesure depuis 1910. Mais le régime de la sécurité de la vieillesse ne fut établi qu'en 1952. Quant à l'assurance-santé, elle est encore plus récente. Il y avait donc énormément à faire, en égard à ces risques, au moment où le Rapport a été écrit.

La dernière partie est consacrée aux besoins familiaux. Comme il se doit, la première place vient aux allocations familiales. Comme l'auteur l'indique lui-même dans son introduction de 1975 , de telles allocations sont importantes pour la cohérence d'un système compréhensif de sécurité sociale. On sait d'ailleurs que le régime d'aide sociale et celui des allocations familiales ont été modifiés au Québec en janvier 1974 de façon à les rendre en un sens, complémentaires l'un de l'autre. Puis sont couverts les besoins des mères, des veuves et des survivants et mêmes les frais funéraires.

Comme on peut le constater dans les lignes qui précèdent, le Rapport Marsh constituait tout un programme pour le temps où il fut produit. Le lecteur peut donc encore une fois évaluer la législation sociale en 1943 et même celle de 1977. Il n'en reste pas moins vrai que ce Rapport ne peut pas intéresser une très forte clientèle, surtout depuis la parution de rapports plus récents et plus complets sur le sujet, tel par exemple le Rapport Castonguay-Nepveu au Québec il y a quelques années. Il s'adresse donc bien sûr au spécialiste et plus particulièrement à l'historien de la législation sociale.

\section{Bertrand BELZILE}

Université Laval

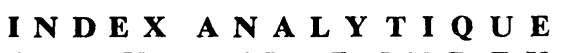

C U M U L A T I V E I N D E X

RELATIONS INDUSTRIELLES

INDUSTRIAL RELATIONS

\author{
Volume 1 - Volume 25 \\ (1945-1970)
}

\title{
Rapport des - Proceedings of CONGRES DES RELATIONS INDUSTRIELLES
}

(1946-1970)

\begin{abstract}
Avant-propos - Forewords; A- Index alphabétique - Subject index; B-1 Index méthodique - Broad Subject Headings Index; Liste des descripteurs génériques - List of generic keywords; B-2 Index méthodique des titres - Broad Subject Heading Index of Titles; C- Index méthodique des ouvrages recensés - Broad Authors of Book Reviews; E- Auteurs des articles et des recensions - Authors of the Articles and Book Reviews; F- Références bibliographiques - Bibliographical References.

Un volume $6 \times 9$, relié, 360 pages

prix : 10.00
\end{abstract}

En vente chez votre libraire ou chez l'éditeur

LES PRESSES DE L'UNIVERSITÉ LAVAL

c.p. 2447, Québec 2, (Qué.) 